\title{
Factores constituyentes identitarios en la maestra de educación infantil
}

Identity Constituent Factors in the Early Childhood Teacher

Artículo de investigación | Research Article

Fecha de recepción: 13 de junio de 2020

Fecha de aceptación: 18 de enero de 2021

Fecha de disponibilidad en línea: septiembre de 2021

doi: 10.11144/Javeriana.m14.fcim

María Soledad Robinson-Seisdedos

maria.robinson@uv.cl

Universidad de Valparaíso, Chile

(D) ORCID: https://orcid.org/0000-0002-2776-177X

José TEJADA-FERNÁNDEZ

jose.tejada@uab.cat

Universitat Autònoma de Barcelona, España

ORCID: https://orcid.org/0000-0001-9044-8826

SílVIA BLANCh-Gelabert

silvia.blanch@uab.cat

Universitat Autònoma de Barcelona, España

(D) ORCID: http://orcid.org/0000-0001-5397-7892

Para citar este artículo | To cite this article

Robinson-Seisdedos, M. S., Tejada-Fernández, J. \& Blanch-Gelabert, S. (2021).

Factores constituyentes identitarios en la maestra de educación infantil. magis,

Revista Internacional de Investigación en Educación, 14, 1-24. doi: 10.11144/

Javeriana.m14.fcim 


\section{Resumen}

El presente artículo analiza los factores claves que inciden en la construcción identitaria de la educadora infantil en sus primeros años de trabajo y en diferentes realidades educativas. La metodología aplicada consistió en un estudio de caso múltiple, con particularidades de tipo biográfico-narrativo, y la recogida de información se realizó a través de relatos de vida, específicamente incidentes críticos y biogramas, junto con entrevistas semiestructuradas y grupos focales. Los resultados dan cuenta de tres grandes dimensiones: biográfica, formación inicial y ejercicio profesional. Los hallazgos permiten concluir que el proceso de construcción identitaria resulta ser complejo, relacional, contextual y variable.

\section{Palabras clave}

Identidad; desarrollo de la carrera; transición a la vida activa; educación de la primera infancia; escuela de párvulos

\section{Abstract}

This article analyzed the key factors that affect the identity construction of the early childhood educator in his/her first years of work and in different educational realities. The methodology consisted of a multiple case study with particularities of a biographical type narrative and the collection of information was carried out through life stories, specifically critical incidents and biograms, together with semi-structured interviews and a focus group. Results showed three large dimensions: biographical, initial education and professional practice. The findings allowed us to conclude that the identity construction process turns out to be complex, relational, contextual and variable.
}

\section{Keywords}

Identity; career development; transition from school to work; early childhood education; nursery school 
Descripción del artículo | Article description

Artículo de investigación científica derivado del trabajo doctoral Construcción de la identidad profesional de la educadora de párvulos principiante en diferentes centros de educación infantil.

\section{Introducción}

Los primeros años de toda persona, reconocidos como una de las fases determinantes para su integral desarrollo (Bueno, 2017; Cantó, 2015), han sido centro de atención en las últimas décadas, modificando de esta manera el reconocimiento por parte de la sociedad a la educación en la primera infancia y a la labor de la maestra en educación infantil (Falabella et al., 2018).

Por ello, la formación inicial para las educadoras de párvulos ha de ser de calidad, en el sentido de las evidencias que señalan la relación entre la formación inicial específica y el desarrollo apropiado de niños y niñas (Pardo \& Alderstein, 2016). Como tal, las diversas tareas que actualmente la maestra de infantil debe llevar a cabo, las variadas realidades en las que lleva a cabo su quehacer pedagógico, la multiplicidad de saberes que requiere conocer y el carácter holístico de su trabajo se traducen en que su labor sea compleja y fundamental (Horm et al., 2013).

Para que la maestra pueda responder a las exigencias de hoy en día, uno de los aspectos esenciales es disponer de una formación profesional inicial pertinente y de calidad, que favorezca en los estudiantes el protagonismo de su proceso de formación (Blanch, 2019). En este sentido, la formación y adquisición de competencias profesionales requiere desarrollar y llevar a cabo de forma significativa las potencialidades del educador y evidenciar los conocimientos necesarios y fundamentales para llevarlos a cabo en el quehacer pedagógico, para que, a partir de su desempeño y la solución de problemas, conflictos o situaciones emergentes vividas en la realidad educativa a la cual pertenece, pueda constatar los conocimientos adquiridos (Blanch \& Tejada, 2017; Tejada et al., 2017).

En cuanto a la licenciatura en educación parvularia, uno de los propósitos fundamentales de desarrollo para las maestras de educación infantil es la promoción en los niños y niñas de sus habilidades, posibilitando aprendizajes con sentido y significado, respetando y respondiendo a las singularidades y particularidades del grupo de párvulos en un contexto educativo específico. Es por esto que estas maestras deben contar con los saberes 
conceptuales, procedimentales y actitudinales, que les permitan desarrollar con niños y niñas aprendizajes de calidad (París et al., 2017; Zapata, 2012).

Por otro lado, los contextos donde se desempeña la educadora juegan un papel fundamental, y deben ser vistos como espacios de interacción constante, de relacionamiento desde lo inter e intrapersonal con diversos actores del proceso educativo, con un fin determinado, en espacios compartidos y con elementos transversales vinculados a los requerimientos desde las políticas públicas (Barrios-Martínez et al., 2019). Es en estos espacios donde se desarrollan situaciones pedagógicas con pares, agentes educativos y comunitarios, en los cuales se van construyendo nuevos aprendizajes, descubriendo y desarrollando de esta manera una identidad propia, sustentada en vivencias anteriores, ya sean profesionales o personales (Ivanova \& Skara-Mincāne, 2016).

Sumado a lo anterior, los primeros años de trabajo en el aula se distinguen por ser un periodo transicional de estudiante a educador, diferenciado de otras etapas por estar condicionado a tensiones y temores originados en la inexperiencia. Sin embargo, es en esta realidad desconocida que el educador va adquiriendo primariamente sus aprendizajes y conocimientos profesionales (Marcelo \& Vaillant, 2013). De acuerdo con lo señalado, esta investigación tiene como principal objetivo analizar el proceso de construcción de la identidad profesional de las maestras de educación infantil principiantes en diferentes centros (públicos, particulares subvencionados y particulares), identificando los factores determinantes de dicho proceso.

Aunque la temática abordada en el presente artículo resulta ser de gran relevancia, las investigaciones en educación infantil referidas principalmente a la construcción identitaria de las educadoras en sus primeros años de trabajo son limitadas. Lo anterior llevó a ampliar la mirada referencialteórica hacia estudios llevados a cabo con maestros de educación primaria y secundaria, teniendo presentes las particularidades referidas al primer nivel educativo, objeto del presente estudio.

\section{Conceptualización}

Actualmente, el concepto de identidad se destaca por ser polisémico. Para Beijaard et al. (2004), es una manifestación de relaciones que se constituyen en la trayectoria profesional y en contextos sociales en la interacción con otros. En este sentido, desde la mirada de los propios educadores se evidencia que la identidad profesional se va construyendo a través de la interacción con los agentes que forman parte del sistema educativo (MadridVivar \& Mayorga-Fernández, 2017). Por lo tanto, se puede establecer que el término de identidad profesional constituye un proceso complejo de 
relaciones, con múltiples significados, que varía en función del punto de vista de los autores y del nivel de significación que les adjudiquen a ciertos factores que la componen (Beijaard et al., 2004; Bolívar, 2016; Day, 2014; Tardif \& Le Vasseur, 2018).

Dentro de las definiciones de identidad profesional destaca lo planteado por Marcelo \& Vaillant (2013), quienes la describen como la definición que realizan los propios educadores de su labor, conformándose así en una construcción del "sí mismo" como profesionales de la educación. Esta construcción se despliega en el ejercicio de la profesión y está determinada por las políticas públicas, las reformas y los centros educativos. Para Bolívar (2016), la identidad es el resultado de la interacción entre las experiencias personales de los educadores y el entorno social, cultural e institucional en el cual se desarrollan habitualmente. Por otro lado, Pillen et al. (2013) señalan que es la respuesta dinámica a constantes transformaciones y diversas vivencias adquiridas durante el transcurso del ejercicio profesional. Con relación a la construcción de la identidad profesional en educación infantil, Vergara (2014) señala que esta se basa en entregar oportunidades pedagógicas pertinentes y de calidad en los párvulos.

En síntesis, y para los efectos de este trabajo, entendemos la identidad profesional, en sentido general, como un producto construido desde la interacción entre un proceso biográfico continuo (individual) y factores relacionales (social-relacional) (Bolívar, 2016). Parte de la noción de sí mismo que porta el profesorado en cuanto enseñante, articulada por las representaciones respecto del trabajo de enseñar, como efecto del contexto de trabajo inscrito en un espacio social (Mórtola, 2006). Conlleva prácticas de interacción compartidas y empieza a conformarse, en algunos casos, antes de tomar la decisión de ser educador, continuando con la formación inicial y consolidándose durante la socialización en el escenario profesional. La identidad no es una característica fija, dado que la autopercepción de los sujetos cambia a medida que transcurre su vida por diferentes etapas, ya sean personales y profesionales, de acuerdo con las condicionantes del contexto laboral, educativo y social (Robinson, 2019).

Es importante señalar que la primera inserción laboral de los profesores principiantes resulta esencial para la construcción y reconstrucción identitaria como educador (Ávalos, 2009). A su vez, constituye una etapa fundamental para decidir su continuidad o retiro como docente, y en este sentido las orientaciones y el acompañamiento recibido pueden ser claves determinantes para su futuro profesional. Cuando la realidad educativa de la cual forma parte inicialmente es compartida por la persona, desde sus creencias y concepciones, su inserción a este contexto puede ser más factible y cómoda (Marcelo, 2011). 
Otro aspecto clave en la construcción identitaria es la reflexión, ya que esta promueve la resignificación de las prácticas pedagógicas al propiciar que el educador pueda construir sus saberes desde la realidad profesional de la que es parte, siendo así protagonista de su aprendizaje (Castellanos \& Yaya, 2013). En línea con lo anterior, este término adquiere especial significado al estar directamente relacionado con la experiencia laboral adquirida en el contexto (Colén et al., 2016).

En este sentido, y en lo que a reflexión se refiere, el 'modelo cebolla' u onion model (Korthagen, 2010) plantea seis niveles, directamente vinculados entre sí, de modo que la búsqueda de estos vínculos se traduce en la reflexión profunda e intencionada de su labor pedagógica como educador. Los niveles de este modelo están referidos a la reflexión respecto al entorno (ambiente), al comportamiento, las competencias, las creencias subyacentes, la identidad y la misión.

\section{Metodología}

Esta investigación se enmarca en el paradigma interpretativo, que supone la comprensión del significado dado por los sujetos participantes a una realidad o contexto del cual ellos forman parte. El enfoque de la investigación es de carácter cualitativo, para poder explorar y comprender el significado atribuido a una problemática social o humana, ya sea de manera grupal o individual (Creswell, 2014; Hernández-Sampieri et al., 2014). En esta investigación, el elemento clave lo constituye la descripción de sucesos desde el punto de vista de las maestras en educación infantil, siendo el propósito de este tipo de estudios conocer en profundidad un hecho o situación determinada, vinculada, en este caso, al campo educativo.

Es necesario señalar que en la presente investigación se han considerado cuestiones éticas, como el resguardo de la información entregada por las participantes, manteniendo su anonimato, junto con la firma de un consentimiento informado, el cual ha permitido transparentar el procedimiento a realizar.

El método desarrollado involucra un estudio de caso múltiple, centrado en la búsqueda sobre un determinado fenómeno valiéndose de diversos casos, los cuales comparten características ya sea por semejanza o discrepancia (Stake, 2013). Se analiza el objeto de estudio en su contexto, considerándolo fundamental tanto para comprobar, ilustrar o construir una teoría o una parte de ella, como por su valor intrínseco, que puede ser transferido a otros casos. El criterio para seleccionar a las participantes 
corresponde a una muestra intencionada de nueve tituladas de licenciatura en educación infantil de una universidad de la región de Valparaíso, Chile, quienes se encuentran en sus tres primeros años de desempeño laboral. Las nueve participantes se titularon durante los años 2014-2015-2016, siendo tres educadoras por cada año y una por curso, que se desempeñan en centros públicos de educación infantil, en centros particulares subvencionados y en jardines infantiles particulares.

Cabe considerar un mínimo apunte al contexto del estudio teniendo presente que, en el 2014, comienza en Chile una reforma educativa, con el fin de mejorar la calidad de la educación pública, eliminar las desigualdades y el beneficio puramente económico, y propiciar la inclusión (MINEDUC, 2016). Los focos en los cuales se orienta esta reforma, en lo que a educación infantil se refiere, son institucionalidad, cobertura y calidad, con el objetivo de propiciar y favorecer una educación con oportunidades de aprendizaje para todas y todos, mejorando, así, el escenario educativo desde la primera infancia. Para su logro, se ha establecido un sistema de aseguramiento de la calidad de la educación para niños y niñas desde los primeros meses hasta los seis años, destacándose, entre otras funciones, la elaboración y actualización de estándares orientadores de desempeño y del marco curricular (MINEDUC, 2016).

En este contexto, la maestra en educación infantil es vista como una agente fundamental y determinante que guía el proceso formativo, organizando y mediando experiencias pedagógicas para toda la comunidad educativa, en beneficio directo de los aprendizajes significativos para niños y niñas.

Como tal, el quehacer pedagógico debe ser asumido, entonces, con compromiso, autonomía y responsabilidad al momento de tomar decisiones orientadas a diseñar, aplicar y evaluar el proceso de enseñanza y aprendizaje. En este sentido, la reflexión, ya sea grupal o individual, contribuye a la resignificación de las prácticas educativas en el desarrollo de la profesión (MINEDUC, 2018).

El propósito de desarrollar esta investigación con tituladas de la misma casa de estudios radica en lo expresado por Bolívar et al. (2005), al plantear que la construcción identitaria de todo educador está condicionada, entre otras, por los aprendizajes obtenidos en la formación inicial. Del mismo modo, y según lo establecido por Huberman (1989), se han seleccionado educadoras que se encuentran en sus tres primeros años de trabajo, ya que este periodo es visto como de ingreso a la carrera, de socialización y conocimiento del contexto pedagógico. La tabla 1 presenta los antecedentes de las maestras en educación infantil en sus primeros años laborales. 
Tabla 1

Caracterización de casos

\begin{tabular}{|c|c|c|c|c|c|c|}
\hline $\begin{array}{l}\text { Institución } \\
\text { ejercicio } \\
\text { profesional }\end{array}$ & Caso & $\begin{array}{l}\text { Año } \\
\text { egreso }\end{array}$ & $\begin{array}{l}\text { Experiencia } \\
\text { profesional }\end{array}$ & Antigüedad & $\begin{array}{l}\text { Nivel } \\
\text { educativo } \\
\text { a cargo }\end{array}$ & $\begin{array}{l}\text { Experiencias } \\
\text { profesionales } \\
\text { anteriores }\end{array}$ \\
\hline \multirow[t]{3}{*}{$\begin{array}{l}\text { Centros } \\
\text { públicos de } \\
\text { educación } \\
\text { infantil }\end{array}$} & 1 & 2014 & $\begin{array}{l}2 \text { años, } \\
6 \text { meses }\end{array}$ & $\begin{array}{l}1 \text { año, } \\
3 \text { meses }\end{array}$ & $\begin{array}{l}\text { Medio } \\
\text { Mayor }\end{array}$ & $\begin{array}{l}\text { Educadora de párvulos } \\
\text { estable y de reemplazo } \\
\text { en centros públicos de } \\
\text { educación infantil }\end{array}$ \\
\hline & 2 & 2015 & $\begin{array}{l}1 \text { año, } \\
7 \text { meses }\end{array}$ & $\begin{array}{l}1 \text { año, } \\
4 \text { meses }\end{array}$ & $\begin{array}{l}1^{\circ} \text { nivel } \\
\text { transición }\end{array}$ & $\begin{array}{l}\text { Educadora de párvulos } \\
\text { de reemplazo en centros } \\
\text { públicos de educación } \\
\text { infantil, } 3 \text { meses. }\end{array}$ \\
\hline & 3 & 2016 & 7 meses & 3 meses & $\begin{array}{l}\text { Nivel medio } \\
\text { menor }\end{array}$ & $\begin{array}{l}\text { Educadora de párvulos } \\
\text { de reemplazo en centros } \\
\text { públicos de educación } \\
\text { infantil, } 4 \text { meses. }\end{array}$ \\
\hline \multirow[t]{3}{*}{$\begin{array}{l}\text { Centros } \\
\text { educativos } \\
\text { particulares } \\
\text { subvencionados }\end{array}$} & 4 & 2014 & $\begin{array}{l}2 \text { años, } \\
9 \text { meses }\end{array}$ & $\begin{array}{l}1 \text { año, } \\
7 \text { meses }\end{array}$ & $\begin{array}{l}2^{\circ} \text { nivel } \\
\text { transición }\end{array}$ & $\begin{array}{l}\text { Educadora de párvulos } \\
\text { en jardines infantiles } \\
\text { particulares, } 1 \text { año, } \\
2 \text { meses. }\end{array}$ \\
\hline & 5 & 2015 & $\begin{array}{l}1 \text { año, } \\
10 \text { meses }\end{array}$ & 5 meses & $\begin{array}{l}1^{\circ} \text { nivel } \\
\text { transición }\end{array}$ & $\begin{array}{l}\text { Educadora de párvulos en } \\
\text { jardín infantil particular } \\
\text { y en centro educativo } \\
\text { particular subvencionado, } \\
1 \text { año, } 5 \text { meses. }\end{array}$ \\
\hline & 6 & 2016 & 9 meses & 5 meses & $\begin{array}{l}2^{\circ} \text { nivel de } \\
\text { transición }\end{array}$ & $\begin{array}{l}\text { Educadora de párvulos } \\
\text { de reemplazo en centros } \\
\text { públicos de educación } \\
\text { infantil, } 4 \text { meses. }\end{array}$ \\
\hline \multirow[t]{3}{*}{$\begin{array}{l}\text { Jardines } \\
\text { infantiles } \\
\text { particulares }\end{array}$} & 7 & 2014 & 7 meses & 5 meses & $\begin{array}{l}\text { Nivel medio } \\
\text { menor }\end{array}$ & $\begin{array}{l}\text { Asistente y educadora de } \\
\text { párvulos de reemplazo, } \\
\text { directora subrogante } \\
\text { en centro público de } \\
\text { educación infantil, } \\
2 \text { meses. }\end{array}$ \\
\hline & 8 & 2015 & $\begin{array}{l}1 \text { año, } \\
10 \text { meses }\end{array}$ & $\begin{array}{l}1 \text { año, } \\
5 \text { meses }\end{array}$ & Heterogéneo & $\begin{array}{l}\text { Educadora de párvulos en } \\
\text { jardín infantil particular, } \\
5 \text { meses. }\end{array}$ \\
\hline & 9 & 2016 & 10 meses & 5 meses & $\begin{array}{l}\text { Nivel medio } \\
\text { menor }\end{array}$ & $\begin{array}{l}\text { Educadora de párvulos } \\
\text { de reemplazo en centros } \\
\text { públicos de educación } \\
\text { infantil, } 5 \text { meses. }\end{array}$ \\
\hline
\end{tabular}

Inicialmente, vinculado al método biográfico narrativo, los instrumentos implementados corresponden a biogramas e incidentes críticos, los cuales, según lo planteado por Bolívar et al. (2001), constituyen los relatos de vida (RV). Estos relatos evidencian a la persona y su identidad (Chase, 2015), siendo una descripción rememorativa que realizan las personas con relación 
a ciertas etapas de su vida (Huchim \& Reyes, 2013; Rivas-Flores, 2014). Estas narraciones se relacionan con vivencias importantes, las cuales responden a cambios significativos en su camino, tanto personal como profesional. Otro de los instrumentos aplicados son las entrevistas semiestructuradas (E), consideradas como una de las principales técnicas que utilizan los investigadores cualitativos para investigar y descubrir realidades múltiples (Stake, 2013). Finalmente se utilizó el grupo focal (GF) para profundizar y obtener información desde la interacción verbal con los participantes en torno al tema investigativo (Álvarez-Gayou, 2012).

En el proceso de validación, los instrumentos fueron sometidos a juicio de expertos a partir de los criterios de univocidad, pertinencia e importancia. Con relación al análisis de la información, entendido como el proceso en el cual el investigador le otorga sentido a los hallazgos que subyacen a lo manifestado por las educadoras que formaron parte de la investigación, y al significado entregado al sistema categorial (deductivo/ apriorístico, inductivo/emergente), los resultados se han clasificado en categorías establecidas según semejanza y diferencia (Bustingorry et al., 2006).

Del mismo modo, dicho análisis se ha efectuado sistemáticamente siguiendo el planteamiento de Miles et al. (2014) relativo a la reducción de datos, que implica organizar y resumir los hallazgos a través del despliegue de la información en códigos, con el soporte de ATLAS.ti 8 (programa cualitativo de análisis de datos).

En la siguiente tabla se evidencia, según su dimensión, la categoría, el significado atribuido por las participantes de la investigación, y de qué manera se establece el sistema categorial: deductivo, desde las investigaciones previas, e inductivo, a partir de los hallazgos en el trabajo de campo. Cabe señalar que hay categorías que responden a ambos sistemas, lo cual ha sido determinado por el instrumento de recogida de información utilizado.

Tabla 2

Tipo y significado de categorías de acuerdo a lo descrito por las participantes

\begin{tabular}{l|l|l|l}
\hline Dimensión & Categoría & Significado & Tipo de categoría \\
\hline Biográfica & $\begin{array}{l}\text { Características } \\
\text { personales }\end{array}$ & $\begin{array}{l}\text { Personalidad y forma de actuar } \\
\text { frente a diferentes vivencias. }\end{array}$ & Inductiva \\
\cline { 2 - 4 } & $\begin{array}{l}\text { Experiencias } \\
\text { de vida }\end{array}$ & $\begin{array}{l}\text { Situaciones desde lo personal antes } \\
\text { del ingreso a la carrera. }\end{array}$ & Deductiva \\
\cline { 2 - 4 } & $\begin{array}{l}\text { Ideas personales } \\
\text { y conflicto de } \\
\text { perspectivas }\end{array}$ & $\begin{array}{l}\text { Cuestionamientos, significados e intereses } \\
\text { antes de ingresar a la formación inicial } \\
\text { en educación parvularia. Pueden ser } \\
\text { modificadas según sus vivencias. }\end{array}$ & \\
\hline
\end{tabular}




\begin{tabular}{|c|c|c|c|}
\hline \multirow[t]{5}{*}{$\begin{array}{l}\text { Formación } \\
\text { inicial }\end{array}$} & $\begin{array}{l}\text { Experiencias } \\
\text { personales }\end{array}$ & $\begin{array}{l}\text { Experiencias personales vividas durante la formación } \\
\text { de pregrado, las que inciden en su proceso } \\
\text { formativo y futuro desarrollo de la profesión. }\end{array}$ & Inductiva \\
\hline & $\begin{array}{l}\text { Formación de } \\
\text { pregrado como } \\
\text { educadora de } \\
\text { párvulos }\end{array}$ & $\begin{array}{l}\text { Aportes entregados por la universidad y la carrera de } \\
\text { educación parvularia donde cursaron sus estudios. }\end{array}$ & Deductiva/Inductiva \\
\hline & $\begin{array}{l}\text { Mejoras en la } \\
\text { formación inicial }\end{array}$ & $\begin{array}{l}\text { Saberes que es necesario abordar en pregrado, con } \\
\text { el propósito de comprenderlos como estudiantes } \\
\text { y aplicarlos como educadoras de párvulos. }\end{array}$ & Deductiva \\
\hline & $\begin{array}{l}\text { Prácticas } \\
\text { pedagógicas } \\
\text { en pregrado }\end{array}$ & $\begin{array}{l}\text { Experiencias significativas preparatorias, vividas } \\
\text { en una comunidad educativa. Estas vivencias } \\
\text { las acercan a su futuro rol de educadoras. }\end{array}$ & Inductiva \\
\hline & $\begin{array}{l}\text { Saberes } \\
\text { relevantes en la } \\
\text { formación inicial }\end{array}$ & $\begin{array}{l}\text { Competencias desarrolladas en la formación inicial, } \\
\text { desde lo conceptual, procedimental y actitudinal. }\end{array}$ & Deductiva \\
\hline \multirow[t]{11}{*}{$\begin{array}{l}\text { Ejercicio } \\
\text { profesional }\end{array}$} & $\begin{array}{l}\text { Apoyo a la } \\
\text { educadora } \\
\text { principiante }\end{array}$ & $\begin{array}{l}\text { Necesidad de contar con educadoras } \\
\text { experimentadas que las orienten en la } \\
\text { resolución de situaciones emergentes. }\end{array}$ & Deductiva/Inductiva \\
\hline & $\begin{array}{l}\text { Aprendizajes } \\
\text { significativos }\end{array}$ & $\begin{array}{l}\text { Experiencias con sentido que han aportado } \\
\text { a la construcción de la identidad profesional } \\
\text { de la educadora de párvulos. }\end{array}$ & Deductiva/Inductiva \\
\hline & $\begin{array}{l}\text { Concepción } \\
\text { propia del rol de } \\
\text { educadora de } \\
\text { párvulos }\end{array}$ & $\begin{array}{l}\text { Definición de su quehacer educativo que } \\
\text { se va construyendo en la comunidad } \\
\text { educativa de la cual forman parte. }\end{array}$ & Deductiva/Inductiva \\
\hline & $\begin{array}{l}\text { Condiciones } \\
\text { laborales }\end{array}$ & $\begin{array}{l}\text { Aspectos vinculados a sus primeras experiencias } \\
\text { de trabajo como educadoras, los cuales } \\
\text { influyen en su quehacer pedagógico. }\end{array}$ & Deductiva/Inductiva \\
\hline & $\begin{array}{l}\text { Contexto } \\
\text { socioeducativo }\end{array}$ & $\begin{array}{l}\text { Diferencias entre diferentes realidades educativas: } \\
\text { públicas, particulares subvencionadas, privadas. }\end{array}$ & Deductiva \\
\hline & $\begin{array}{l}\text { Significado de } \\
\text { la reflexión en } \\
\text { el quehacer } \\
\text { pedagógico }\end{array}$ & $\begin{array}{l}\text { Sentido de analizar y evaluar las prácticas } \\
\text { pedagógicas, ya sea individual o grupalmente, con } \\
\text { el propósito de mejorar y resignificar su quehacer. }\end{array}$ & Deductiva \\
\hline & $\begin{array}{l}\text { Situaciones } \\
\text { laborales } \\
\text { complejas }\end{array}$ & $\begin{array}{l}\text { Experiencias difíciles de enfrentar, vividas con el } \\
\text { equipo técnico pedagógico, niñas y niños, y familias. }\end{array}$ & Inductiva \\
\hline & $\begin{array}{l}\text { Tipo de } \\
\text { educadora }\end{array}$ & $\begin{array}{l}\text { Características de la educadora de } \\
\text { párvulos para llevar a cabo su rol. }\end{array}$ & Inductiva \\
\hline & $\begin{array}{l}\text { Trabajo en } \\
\text { equipo y } \\
\text { colaborativo }\end{array}$ & $\begin{array}{l}\text { Significado y relevancia que le otorgan al } \\
\text { trabajo con otros profesionales y actores de la } \\
\text { comunidad educativa a la cual pertenecen. }\end{array}$ & Deductiva/Inductiva \\
\hline & $\begin{array}{l}\text { Trabajo } \\
\text { pedagógico }\end{array}$ & $\begin{array}{l}\text { Prácticas desarrolladas en la comunidad educativa } \\
\text { con diferentes actores del proceso educativo. }\end{array}$ & Deductiva/Inductiva \\
\hline & $\begin{array}{l}\text { Valoración del } \\
\text { trabajo realizado } \\
\text { por la educadora }\end{array}$ & $\begin{array}{l}\text { Reconocimiento social entregado a la educadora de } \\
\text { párvulos en lo que a su labor pedagógica se refiere. }\end{array}$ & Inductiva \\
\hline
\end{tabular}

Nota. En esta tabla se presentan todas las categorías según su dimensión, el significado que le entregan las educadoras principiantes y el tipo de categoría. 


\section{Resultados}

Los resultados muestran, con mayor detalle, los aspectos claves que las participantes expresan como más influyentes en la construcción de su identidad como maestras. En este estudio, las tres macrocategorías o dimensiones que se evidencian son: biográfica, formación inicial y ejercicio profesional. Dentro de estas se concretan categorías y subcategorías referidas a la información dada a conocer por las participantes del estudio. Cabe notar que dichas dimensiones se basan en las investigaciones de Ávalos (2016), quien señala que los aspectos determinantes en la construcción identitaria del educador se sustentan en la historia de vida, la formación de pregrado y el ejercicio profesional, de acuerdo con la realidad educativa de la cual forma parte la educadora.

En la tabla 3 se resumen todas las categorías encontradas y su frecuencia, organizadas a partir de los procedimientos utilizados en la recogida de información. En el cuadro se diferencian, además, las categorías apriorísticas y las emergentes resultantes. Las categorías apriorísticas provienen de la revisión bibliográfica y de estudios previos revisados en la literatura científica. En cambio, las categorías emergentes surgen a partir del trabajo de campo. El estudio que se presenta es parte de una investigación cuyo propósito ha sido analizar el proceso constituyente identitario de las maestras en educación infantil en sus inicios laborales y en contextos de trabajo diversos. Sin embargo, en este artículo se incide en aquellos aspectos más relevantes que determinan dicho proceso. De acuerdo con lo anterior, en la tabla se representan las categorías más significativas en gris.

Tabla 3

Resumen de categorías y su fundamentación según dimensiones

\begin{tabular}{|c|c|c|c|c|c|c|c|}
\hline \multirow{3}{*}{ Dimensión } & \multirow{3}{*}{ Categoría } & \multicolumn{6}{|c|}{ Instrumento } \\
\hline & & \multicolumn{2}{|c|}{$\begin{array}{c}\text { Relatos de vida } \\
\text { (Biogramas } \\
\text { e incidentes } \\
\text { críticos) }\end{array}$} & \multicolumn{2}{|c|}{$\begin{array}{l}\text { Entrevistas } \\
\text { en } \\
\text { profundidad }\end{array}$} & \multicolumn{2}{|c|}{ Grupo focal } \\
\hline & & $\mathbf{F}$ & TC & $\mathbf{F}$ & TC & $\mathbf{F}$ & TC \\
\hline \multirow{3}{*}{ Biográfica } & Características personales & & & 12 & $\mathrm{E}$ & & \\
\hline & Experiencias de vida & 60 & A & 25 & A & & \\
\hline & Ideas personales y conflictos de perspectivas & 43 & $\mathrm{E}$ & 23 & A & & \\
\hline \multirow{5}{*}{$\begin{array}{l}\text { Formación } \\
\text { inicial }\end{array}$} & Experiencias personales & 25 & $\mathrm{E}$ & 9 & $\mathrm{E}$ & & \\
\hline & $\begin{array}{l}\text { Formación de pregrado como educadora } \\
\text { de párvulos }\end{array}$ & 47 & $\mathrm{E}$ & 88 & A & 4 & $\mathrm{E}$ \\
\hline & Mejoras en la formación inicial & & & 33 & A & & \\
\hline & Prácticas pedagógicas en pregrado & 56 & $\mathrm{E}$ & 39 & $\mathrm{E}$ & & \\
\hline & Saberes relevantes en la formación inicial & & & 24 & $A$ & 13 & $A$ \\
\hline
\end{tabular}




\begin{tabular}{|c|c|c|c|c|c|c|c|}
\hline \multirow{11}{*}{$\begin{array}{l}\text { Ejercicio } \\
\text { profesional }\end{array}$} & Apoyo a la educadora principiante & & & 10 & $E$ & 37 & A \\
\hline & Aprendizajes significativos & 80 & $A$ & 49 & $E$ & 29 & A \\
\hline & $\begin{array}{l}\text { Concepción propia del rol de educadora } \\
\text { de párvulos }\end{array}$ & 13 & A & 86 & A & 9 & $E$ \\
\hline & Condiciones laborales & 22 & $\mathrm{E}$ & 28 & $E$ & 24 & A \\
\hline & Contexto socioeducativo & 11 & $E$ & 183 & A & 41 & A \\
\hline & $\begin{array}{l}\text { Significado de la reflexión en } \\
\text { el quehacer pedagógico }\end{array}$ & & & & & 35 & A \\
\hline & Situaciones laborales complejas & 28 & $\mathrm{E}$ & & & 15 & $E$ \\
\hline & Tipo de educadora & & & 33 & $\mathrm{E}$ & & \\
\hline & Trabajo en equipo y colaborativo & 11 & $\mathrm{E}$ & 94 & A & 27 & $\mathrm{E}$ \\
\hline & Trabajo pedagógico & 11 & $E$ & 94 & A & 16 & $E$ \\
\hline & $\begin{array}{l}\text { Valoración del trabajo realizado } \\
\text { por la educadora }\end{array}$ & 9 & $E$ & 23 & $E$ & 10 & $E$ \\
\hline
\end{tabular}

$\mathrm{F}=$ Frecuencia

TC= Tipo de categoría

$\mathrm{E}=$ Emergente

A $=$ Apriorística

Nota. La tabla evidencia en color gris las categorías más significativas de acuerdo

a su recurrencia y relevancia, según los instrumentos utilizados en la recogida de información.

A continuación, y con relación a lo resaltado en gris en la tabla 3, se presentan las categorías, según su dimensión, seleccionadas como determinantes y claves del proceso de construcción identitaria, de acuerdo con el instrumento de recogida de información aplicado, señalando el significado atribuido por las educadoras participantes del estudio e incorporando evidencias.

\section{Dimensión: Biográfica}

\section{Experiencias de vida}

Los hallazgos para esta categoría dan cuenta de aquellas vivencias que tuvieron las maestras antes de cursar sus estudios en educación infantil. Estas experiencias influyeron en el cambio de perspectiva con relación a ciertos aspectos de su vida y, de algún modo, incidieron en su futuro personal y profesional.

[...] este año viví una de las pruebas más difíciles de mi vida, que fue la muerte de mi padre. Este acontecimiento, junto con el nacimiento de mi hija, me hicieron replantearme todo, y me dieron la motivación de encontrar alguna carrera que se ajustara con mi personalidad. (RV9)

Las subcategorías son: emocionalidad desde lo personal, personas significativas, significancia desde lo personal, situaciones laborales de 
conflicto, término de una etapa, tipo de experiencia personal, reflexión como crecimiento personal, ser madres y situaciones laborales de conflicto. En las evidencias que arrojan los relatos de vida, se establece que las tres últimas subcategorías presentan mayor recurrencia, las cuales, de acuerdo al significado que les entregan las participantes de la investigación, se entienden como:

Reflexión como crecimiento personal: Es considerada por las educadoras participantes como un medio para resignificar su mirada de mundo y mejorar en su quehacer pedagógico. Las reflexiones efectuadas a partir de las situaciones vividas propiciaron en ellas la toma de decisiones desde lo personal a lo profesional.

Lo más significativo fue abrir los ojos y madurar respecto a lo que quería para mi vida. Que si bien aún no tenía claro qué estudiar, sabía que algo que tuviese que ver con escritorios y oficinas no sería. (RV3)

El asunto significó que me cuestionara todo lo que quería y soñaba hacer: ¿seré buena para esto?, ¿serviré para esto?, ¿Dios quiere otra cosa para mi vida? Mis papás me dieron la oportunidad de hacer un preuniversitario y dar la prueba otra vez. Ese año fue un tiempo para cuestionarme lo que quería hacer en mi vida y para lo que era buena. (RV4)

Ser madre: Este acontecimiento es visto por las participantes como una etapa de madurez y de gran compromiso, por lo que el estudiar educación infantil después de tener a su hijo ha propiciado en ellas comprender con mayor sentido y significado el rol de ser madres, y descubrir las etapas de desarrollo de la primera infancia desde los contenidos vistos en las asignaturas.

Las emociones que experimenté no solo fueron de felicidad; también experimenté temor, tenía miedo de no hacer lo mejor de mí, o no responder como madre a esa pequeña criatura que de la nada estaba en mis brazos. (RV9)

Pero poco a poco comencé a canalizar mis energías solo en salir adelante, sabía que podía, nunca me había costado mucho estudiar, solo que ahora tenía claro que debía colocar el doble de esfuerzo. (RV2)

Trabajo con grupo de niños: Las participantes lo caracterizan por su participación como monitoras en actividades, asistiendo a hogares de niños o desarrollando programas infantiles en grupos de iglesia. Lo anterior se vincula a lo expresado por Ávalos (2016), al plantear que las vivencias relacionadas al trabajo con grupo de niños o jóvenes, previo a sus estudios 
de pregrado, es una de las particularidades que guían a los estudiantes a decidirse por la educación como profesión.

Cerca de mis 16 años comencé a participar de un grupo de jóvenes voluntarios que trabajan con niños de 4 a 12 años en situación vulnerable. (RV6)

Llevaba al menos 6 años trabajando con niños y además ingresé a una fundación para lo mismo; por ende, el incidente me permitió mirar más allá de lo que quería en el momento y darme cuenta de que mis habilidades y mi pasión iban por otro camino que no era el periodismo. (RV8)

\section{Dimensión: Formación inicial}

Formación de pregrado como educadora de párvulos

El significado atribuido por las educadoras que formaron parte de esta investigación se vincula con las características, aportes y aspectos relevantes vividos como estudiantes de la universidad donde cursaron sus estudios, los cuales han contribuido y orientado su desarrollo profesional en el campo laboral.

[...] aparte que es una universidad súper exigente [...] uno se va exigiendo más, entonces cuando uno ya se va comparando con otras colegas, uno sí es más meticuloso, es más organizado, como que es más cuadradito en ese sentido, siempre tener las cosas al día y seguir estudiando, perfeccionándose, eso es como que siempre ha marcado, es como un sello siento yo, el sello de ir perfeccionándome. (E1)

Dentro de las subcategorías, se establecen: grado de dificultad de los estudios cursados, importancia de la universidad y la carrera, ingreso a la carrera, valoración de la carrera por docentes de otras áreas, contenidos entregados y referentes en la formación de pregrado. Estas dos últimas subcategorías resultan ser de mayor relevancia para las maestras participantes, las cuales implican:

Contenidos entregados: A partir de las asignaturas abordadas, pudieron evidenciar que la licenciatura de educación infantil alcanza mayor profundidad de lo que imaginaban, dado que los contenidos abordados se relacionan de manera directa con las acciones a llevar a cabo como futuras educadoras de párvulos, los cuales en la actualidad han resultado ser recursos significativos y necesarios en su quehacer pedagógico.

[...] después, ya en la carrera cuando fui conociendo los distintos temas, me fui dando cuenta que era mucho más profundo, no era tan fácil, sino que tenía muchos temas dentro de educación de la salud, dentro de lo psicológico, dentro del desarrollo [...]. (E2) 
Todo lo que me enseñaron en la universidad, esto del pensamiento crítico, hacer pensar a los niños, del buen trato, la mediación, todo era lo que a mí me enseñaron, entonces ahí yo lo puse en práctica. (E9)

Referentes en la formación de pregrado: Las integrantes de este estudio destacan la relevancia que tuvo en su formación de pregrado contar con educadores-referentes. En este sentido, le otorgan importancia a las relaciones cercanas que mantenían con profesores, en la medida en que los docentes se transforman en modelos a seguir en el ámbito profesional.

[...] bastante, sobre todo una profesora. No me imagino el hecho de haber estudiado en otra universidad sin esas profes, iporque nos marcan tanto!, pero en la cercanía, en el hecho de la confianza, en decir que "ya, cometiste un error, pero mira lo puedes ir mejorando", o sea, esa cercanía dudo que se haya dado en otra universidad con otras profesoras. (E5)

[...] yo creo que se fue dando dentro de la formación, sí, netamente dentro de las profesoras, de ir viendo cómo ellas iban investigando [...] a uno también les daban ganas de seguir, de seguir más allá. (E1)

\section{Dimensión: Ejercicio profesional}

\section{Contexto socioeducativo}

Las educadoras investigadas hacen referencia al ambiente y al espacio pedagógico en el que se desarrollan como profesionales. En estas realidades se identifican aspectos diferenciadores con relación a otros centros educativos, dado que el grado de conocimiento que posea la educadora de su realidad es esencial para llevar a cabo propuestas pedagógicas con las familias. Por otro lado, su desempeño está condicionado por aspectos como el nivel de satisfacción laboral y el comprender y compartir el sello institucional. En este sentido, es en la inserción laboral en sus primeros años de trabajo y en las exigencias institucionales que la maestra en educación infantil va construyendo su identidad profesional.

Las subcategorías evidenciadas desde los hallazgos son: características de las familias, grado de satisfacción profesional, nivel de conocimiento del contexto y característica de la institución donde trabaja, siendo esta última la de mayor recurrencia y relevancia.

Características de la institución donde trabaja: Es comprendida como la forma de funcionamiento del centro educativo en el que la educadora lleva a cabo su labor pedagógica en los contextos en estudio (centros públicos en educación infantil, particulares subvencionados y jardines infantiles particulares). 
Es una educación que yo siento que se preocupa por el bienestar de los niños y sobre todo por la calidad, porque siempre está en una constante formación para que las funcionarias se capaciten, [...] que también abarca no solo a niños con vulnerabilidad social, sino que también ahora tiende a la universalización, dentro de la misión de la institución, más a la inclusión. (E1)

Es que claro, ahora yo me lo podría cuestionar en el sentido de por qué tienen que hacerles una prueba a niños tan pequeños [...] (E4)

Lucrativa, totalmente, no he logrado ver de los dueños o la directora una real importancia en que le interesen los niños o que aprendan. Siento que son números y obviamente es súper válido, yo no lo voy a juzgar ni nada de eso, es un jardín privado, yo me fui a meter allá y no los voy a juzgar por eso. (E9)

\section{Apoyo a la educadora principiante}

Las participantes del estudio subrayan la falta de estrategias para la resolución de situaciones emergentes o de conflicto manifestadas en el quehacer pedagógico, ya que dada su inexperiencia no cuentan con los recursos ni con las competencias requeridas para resolverlas. Expresan, además, que estas necesidades hacen referencia a las orientaciones o acompañamiento que deberían recibir del equipo educativo, específicamente de otra educadora con mayor experiencia en el contexto.

Yo la necesidad de apoyo principal que tenía en mi trabajo era la empatía, yo sentía que mis colegas, mis compañeras de trabajo eran muy poco empáticas, y como que todos me culpaban a mí porque no me resultaba el trabajo con mis agentes educativas en sala. (GF3)

Por otro lado, para las educadoras es fundamental contar con el respaldo del equipo directivo y pedagógico. Las subcategorías en este aspecto se relacionan con:

Requerimientos de la educadora: De acuerdo a lo vivido por las educadoras participantes del estudio, estos requerimientos tienen que ver con aspectos que necesitan en sus primeros años de inserción laboral, los cuales contribuirán a que su trabajo se desarrolle de manera más integral. Entre ellos resalta la importancia de contar con equipos empáticos y de trabajar con especialistas para el diagnóstico de niños y niñas con necesidades educativas especiales, estableciendo de esta manera redes de apoyo, especialmente en las instituciones educativas particulares subvencionadas y los jardines infantiles particulares. 
[...] porque este año, por ejemplo, tengo un caso particular de una niña que le cuesta mucho el aprendizaje, y como que yo ya intenté todos los métodos que yo conozco y no sé detectarle si es que tiene algo más, entonces, no sé qué hacer. (GF4)

Respaldo desde la institución: Esta subcategoría la visualizan bajo dos miradas. Por una parte, las acciones llevadas a cabo por el centro educativo, especialmente en las instituciones del Estado, con el propósito de entregar los apoyos pertinentes para que la maestra en educación infantil pueda gestionar de manera adecuada en la realidad pedagógica a la cual pertenece.

Es súper importante lo de las redes de apoyo; de hecho yo en mi jardín tengo un grupo, una dupla SENADI que va a trabajar junto con la educadora diferencial [...] ellas van a trabajar con los chicos que tenemos con necesidades educativas especiales. (GF1)

Por otro lado, las maestras se refieren a esta subcategoría como el soporte que no es otorgado por la institución educativa, afectando esto en su inserción laboral; lo anterior es descrito por educadoras de centros particulares subvencionados y jardines infantiles particulares.

Lo otro también es que en los jardines particulares o colegios las capacitaciones son más bien como personales. Por ejemplo, a uno le pueden dar los datos de capacitaciones y todo, pero es como el dato, pero no existe mucho como el "hay una capacitación para las educadoras en tal lugar". Ellos te pueden dar las facilidades, pero no te dan la oportunidad concreta, entonces, yo creo que eso falta. (GF4)

\section{Discusión de resultados y conclusiones}

El principal propósito que tienen los hallazgos dados a conocer consiste en comprender y extender el conocimiento del proceso constituyente identitario en la maestra de educación infantil, en sus primeros años de ejercicio laboral y en contextos educativos chilenos diversos. En cuanto a identificar los factores que determinan el proceso de construcción identitaria, se evidencian:

- Situaciones significativas vividas antes de realizar estudios de licenciatura en educación parvularia.

- Experiencias en la licenciatura en educación parvularia, desde lo personal y profesional.

- Eventos vivenciados en el ejercicio de la profesión. 
Del mismo modo, se establece una directa relación entre el contexto de práctica en la interacción con agentes educativos y comunitarios y la construcción identitaria, al tener puntos en común, tales como concepciones, ideas y una mirada de infancia compartida. En este sentido, y según lo expresado por Ávalos \& Sevilla (2010), estas realidades laborales establecen la manera como los educadores se identifican, definen y orientan sus fines y propósitos, tanto profesionales como personales.

En los primeros años de trabajo de la educadora, de acuerdo a las experiencias vividas y a las características de la institución de la cual es parte, va conociendo y comprendiendo la realidad educativa, desarrollándose como profesional y construyendo, así, su identidad como maestra. En línea con lo anterior, Bolívar (2016) señala que la identidad de los educadores se vincula con las prácticas pedagógicas y el contexto en el que se llevan a cabo, convirtiéndose lo vivido en una experiencia social construida y reconstruida a partir de realidades diversas.

En lo referido a las particularidades institucionales en que se desempeñan, estas se relacionan con las formas de funcionamiento del contexto al cual pertenecen, haciendo referencia a:

Centros públicos en educación infantil: El objetivo principal se centra en propiciar el bienestar del niño y la niña, contribuyendo, además, en la entrega de una educación de calidad. La relación con las familias es cercana, reconociéndolas como el primer agente educativo de sus hijos (MINEDUC, 2018). Con relación al trabajo en equipo, este es reconocido como esencial para el logro de metas y para enriquecer las prácticas educativas. En este sentido, se llevan a cabo de manera frecuente cursos de formación y capacitaciones, que contribuyen a mejorar el quehacer pedagógico, lo cual redunda en beneficio de niños y niñas y de toda la comunidad educativa.

Los procesos reflexivos son de gran importancia, ya que posibilitan resignificar lo aprendido y vivido, en beneficio de la propia educadora y del equipo pedagógico, unificando criterios de organización y trabajo en conjunto.

Centros educativos particulares subvencionados: Se le otorga gran relevancia al resultado más que al proceso de aprendizaje, de modo que las prácticas pedagógicas se caracterizan por ser conductistas. A pesar de que las educadoras principiantes manifiestan la importancia de involucrar a las familias en el proceso educativo, y lo fundamental de la reflexión para crecer como profesionales (Castellanos \& Yaya, 2013; Colén et al., 2016; Robinson et al., 2018), estas acciones no son llevadas a cabo porque no se encuentran dentro de los requerimientos institucionales.

Jardines infantiles particulares: Realidad lucrativa, que se financia por las mensualidades que pagan las familias, por lo que prima que se sientan 
cómodos en la institución. El método de trabajo es libre y no se evidencia la reflexión como proceso para el mejoramiento de las prácticas pedagógicas y el trabajo en equipo. Con respecto a las remuneraciones, estas se caracterizan por ser bajas, considerando las extensas jornadas laborales que deben cumplir.

Como señalan Ávalos \& Sevilla (2010), todo educador corrobora la definición que tiene de sí mismo como profesional en sus primeras experiencias de trabajo en una comunidad educativa específica, lo que propicia la construcción identitaria como maestro. En este sentido, el acompañamiento, respaldo y orientación que reciba de la realidad educativa de la cual forma parte es fundamental para resolver situaciones emergentes vividas en el día a día en la práctica de su profesión (Alarcón et al., 2015).

De acuerdo con lo anterior, se plantean algunas proyecciones o propuestas de mejoras orientadas a propiciar aprendizajes en las educadoras de párvulos principiantes, en beneficio de su profesionalización, su desarrollo profesional y la mejora de la calidad de la educación parvularia (Robinson, 2019). Estas proyecciones se refieren a:

Acompañamiento pedagógico-emocional: Cabe señalar que para las maestras en educación infantil lo esencial es poder contar con un equipo que apoye su trabajo y les dé soporte emocional, evidenciando la empatía como una práctica cotidiana, conducente a mejorar los ambientes laborales y el trabajo colaborativo.

Redes de trabajo: Se requiere establecer redes de apoyo y en equipo, con el fin de trabajar de manera interdisciplinaria, oportuna y pertinente, y que el centro cuente con especialistas para diagnosticar necesidades educativas especiales en los párvulos.

Reflexión sistemática, intencionada, en profundidad y con autocrítica: Tal ejercicio debería llevarse a cabo sistemáticamente, teniendo en cuenta los niveles que involucra (Korthagen, 2010). De este modo, las educadoras de párvulos podrán ir construyendo su identidad profesional con mayor consciencia y consistencia del proceso, lo cual favorecerá su desarrollo profesional y personal, al contribuir la reflexión en los aprendizajes para la vida, siempre y cuando esta resulte ser significativa (Robinson, 2019).

En vista de los resultados, podemos establecer que la construcción identitaria es producto de un sinnúmero de elementos (Bajardi \& ÁlvarezRodríguez, 2015; Cantón, 2018), cuya relación entre lo profesional y lo personal no se puede dividir (Sancho et al., 2014). Por otro lado, la educadora de párvulos realiza su trabajo en realidades culturales y sociales en permanente contacto, y en relación con el equipo pedagógico, familias, niños y niñas, entre otros, lo cual favorece la comprensión del contexto, y el autoconcepto, para llegar al saber profesional (Orozco, 2017). 
Finalmente, referido a las limitaciones del estudio, y de acuerdo con lo ya expresado, la bibliografía vinculada a la construcción identitaria de la educadora de párvulos principiante es escasa, por lo cual se buscó, complementó y construyó teoría a partir de investigaciones de la temática abordada para educadores de educación primaria y secundaria. En línea con lo anterior, y desde la prospectiva de esta investigación, se pretende seguir indagando en temáticas vinculadas a esta área, principalmente en lo que refiere a los conflictos identitarios del contexto laboral-pedagógico, la transición de estudiante a educadora, y la identidad profesional en la interacción con diferentes actores del proceso educativo.

No obstante, es importante señalar que desde los hallazgos y aportes del presente estudio, los factores constituyentes identitarios de la educadora de párvulos principiante involucran las características personales, esto es, la personalidad, las experiencias personales vividas durante la etapa de formación inicial en educación infantil y las prácticas pedagógicas de pregrado, las cuales las acercaron a la realidad para su futuro laboral. Desde el ejercicio profesional, destacan las situaciones laborales complejas, referidas principalmente a las dificultades para el trabajo en equipo, las diferencias de opinión con las familias y el comportamiento de niños y niñas; del mismo modo se cuentan las características propias como educadora, que permiten desarrollar su rol pedagógico, y la valoración o reconocimiento social entregado por otros con relación a su quehacer profesional.

\section{Agradecimientos}

Este artículo se desarrolla en el marco de la tesis doctoral que lleva por nombre Construcción de la identidad profesional de la educadora de párvulos principiante en diferentes centros de educación infantil. Estudio de caso, llevada a cabo con el financiamiento del Programa de Formación de Capital Humano Avanzado - Becas Chile 2015, de la Comisión Nacional Científica y Tecnológica (CONICYT).

\section{Sobre los autores}

María Soledad Robinson-Seisdedos es educadora de párvulos, docente universitaria, especialista en Educación Diferenciada (mención Trastornos Específicos del Aprendizaje), magíster en Educación Inicial (mención Didáctica del Lenguaje y Matemáticas), doctora en Educación. Docente y educadora guía de la Escuela de Educación Parvularia, perteneciente a la Facultad de Medicina de la Universidad de Valparaíso, Chile. 
José Tejada-Fernández es doctor en Ciencias de la Educación, catedrático de Didáctica y Organización Escolar en el Departamento de Pedagogía Aplicada de la Universitat Autònoma de Barcelona, España. Especialista en evaluación de programas, innovación formativa, formación para el trabajo y formación de profesionales de la educación.

Sílvia Blanch-Gelabert es psicóloga clínica, doctora en Psicología de la Educación, profesora agregada del Departamento de Psicología Básica Evolutiva y de la Educación de la Universitat Autònoma de Barcelona, España. Especialista en Educación Infantil, Familia y Formación Permanente a Profesionales de la Educación.

\section{Referencias bibliográficas}

Alarcón, J., Castro, M., Frites, C. \& Gajardo, C. (2015). Desafíos de la educación preescolar en Chile: Ampliar la cobertura, mejorar la calidad y evitar el acoplamiento. Estudios Pedagógicos, XL/(2), 287-303. http://dx.doi. org/10.4067/S0718-07052015000200017

Álvarez-Gayou, J. (2012). Cómo hacer investigación cualitativa. Fundamentos y metodología. Paidós.

Ávalos, B. (2009). La inserción profesional de los docentes. Profesorado. Revista Currículum y Formación del Profesorado, 13(1), 43-59. https://www.ugr. es/ recfpro/rev131ART3.pdf

Ávalos, B. (2016). Learning from research on beginning teachers. En J. Loughran \& M. Hamilton (Eds.), International handbook of teacher education (pp. 487-522). Springer.

Ávalos, B. \& Sevilla, A. (2010). La construcción de la identidad profesional en los primeros años de docencia: evidencia desde la investigación. Centro de Investigación Avanzada en Educación. Universidad de Chile.

Bajardi, A. \& Álvarez-Rodríguez, D. (2015). El cuerpo docente y los procesos de configuración de la identidad profesional. Opción, 31(5), 111-129. https:// dialnet.unirioja.es/servlet/articulo?codigo $=5963080$

Barrios-Martínez, D. M., Zuluaga-Ocampo, Z. P., García-Cepero, M. C., GómezHernández, F. A., Santamaría, A., Castro-Fajardo, L. E. \& Sánchez-Vallejo, A. (2019). Comunidades de práctica como marco comprensivo del talento docente. Magis, Revista Internacional en Educación, 11(23), 75-94. https:// doi.org/10.11144/Javeriana.m11-23.cpmc

Beijaard, D., Meijer, P. \& Verloop, N. (2004). Reconsidering research on teachers's professional identity. Teaching and Teacher Education, 20, 107-128. https:// doi.org/10.1016/j.tate.2003.07.001

Blanch, S. (2019). La formación de maestros de educación infantil en la Universitat Autònoma de Barcelona. Revista entreideias, 8(2), 181-203. https://doi. org/10.9771/re.v8i2.28950

Blanch, S. \& Tejada, J. (2017). El trabajo en equipo cooperativo, estrategia para la adquisición y el desarrollo de competencias genéricas y específicas en la asignatura de Inclusión Educativa en el Grado de Educación Infantil. En M. Pérez Ferra \& J. Rodríguez Pulido (Coords.), Buenas prácticas docentes del profesorado universitario (pp. 47-66). Octaedro. 
Bolívar, A. (2016). Reconstruir la identidad profesional docente en la sociedad del conocimiento. En J. Valle \& J. Manso (Dirs.), La 'cuestión docente', nuevas perspectivas (pp. 15-29). Narcea.

Bolívar, A., Domingo, J. \& Fernández, M. (2001). La investigación biográficonarrativa en educación. Enfoque y metodología. La Muralla.

Bolívar, A., Fernández, M. \& Molina E. (2005). Investigar la identidad profesional del profesorado: Una triangulación secuencial. Forum: Qualitative Social Research, 6(1), Art. 12. https://doi.org/10.17169/fqs-6.1.516

Bueno, D. (2017). Neurociencias para educadores. Octaedro.

Bustingorry, S., Sánchez, I. \& Ibáñez, F. (2006). Investigación cualitativa en educación. Hacia la generación de teoría a través del proceso analítico. Estudios Pedagógicos, XXXII(1), 119-133. http://dx.doi.org/10.4067/S071807052006000100007

Cantó, J. (2015). Resultados de la implementación de la neurodidáctica en las aulas de educación infantil. Opción: Revista de Ciencias Humanas y Sociales, 31(5), 189-199. https://www.redalyc.org/articulo.oa?id=31045570011

Cantón, I. (2018). La calidad identitaria del profesorado a través de su satisfacción profesional. En M. Tardif, \& I. Cantón (Eds.), Identidad profesional docente (pp. 33-44). Narcea.

Castellanos, S. \& Yaya, R. (2013). La reflexión docente y la construcción del conocimiento: una experiencia desde la práctica. Sinéctica, (41), 1-18.

Colén, Ma . T., Jarauta, B. \& Borrasca, C. (2016). El aprendizaje reflexivo en la formación inicial de maestros/as: de la experiencia a la integración y síntesis de los contenidos. Revista Complutense de Educación, 27(2), 179-198. https:// doi.org/10.5209/rev_RCED.2016.v27.n1.45679

Chase, S. (2015). Investigación narrativa. Multiplicidad de enfoques, perspectivas y voces. En N. Denzin \& Y. Lincoln (Coords.), Manual de investigación cualitativa. Volumen IV. Métodos de recolección y análisis de datos (pp. 58-112). Gedisa S.A.

Creswell, J. (2014). Research design. Qualitative, quantitative and mixed methods approaches. SAGE.

Day, C. (2014). Pasión por enseñar. La identidad personal y profesional del docente y sus valores. Narcea.

Falabella, A., Cortázar, A., Godoy, F. \& Romo, F. (2018). Sistemas de aseguramiento de la calidad en educación inicial: lecciones desde la experiencia internacional. Gestión y Política Educativa, XXVII(2), 309-340. http://www.scielo. org.mx/scielo.php?script=sci_arttext\&pid=S1405-10792018000200309

Horm, D., Hyson, M. \& Winston, P. (2013). Research on early childhood teacher education: Evidence from three domains and recommendations for moving forward. Journal of Early Childhood Teacher Education, 34, 95-112.

Hernández-Sampieri, R., Fernández, C. \& Baptista-Lucio, M. (2014). Metodología de la Investigación ( $6^{\circ}$ ed.). McGraw-Hill.

Huberman, M. (1989). The professional life cycle of teachers. Teachers College Record, 91(1), 31-57. https://eric.ed.gov/?id=EJ398425

Huchim, D. \& Reyes, R. (2013). La investigación biográfico-narrativa. Una alternativa para el estudio de los docentes. Actualidades Investigativas en Educación, 13(3), 1-27. https://www.redalyc.org/pdf/447/44729878019.pdf 
Ivanova, I. \& Skara-Mincāne, R. (2016). Development of professional identity during teacher's practice. ELSEVIER. Procedia - Social and Behavioral Sciences, (232), 529-536. https://doi.org/10.1016/j.sbspro.2016.10.073

Korthagen, F. (2010). La práctica, la teoría y la persona en la formación del profesorado. Revista Universitaria de Formación del Profesorado, 68(24,2), 83101. https://dialnet.unirioja.es/servlet/articulo?codigo $=3276048$

Madrid-Vivar, D. \& Mayorga-Fernández, M. J. (2017). Construcción y reconstrucción de la identidad de los futuros docentes a través de sus autobiografías académicas. Revista Complutense de Educación, 28(2), 375-389. https:// dialnet.unirioja.es/servlet/articulo?codigo $=6359426$

Marcelo, C. (2011). Políticas de inserción en la docencia: de eslabón perdido a puente para el desarrollo profesional docente. Programa de Promoción de la Reforma Educativa en América Latina y el Caribe (PREAL).

Marcelo, C. \& Vaillant, D. (2013). Desarrollo profesional docente ¿Cómo se aprende a enseñar? Narcea S.A. Ediciones.

Miles, M. B., Huberman, A. M. \& Saldaña, J. (2014). Qualitative Data Analysis. A Methods Sourcebook ( $3^{\mathrm{a}}$ ed.). SAGE.

Ministerio de Educación. (2016). La Reforma educacional está en marcha. Cuenta pública 2015. Subsecretaría de Educación. MINEDUC.

Ministerio de Educación. (2018). Bases curriculares de la educación parvularia. MINEDUC.

Mórtola, G. (2006). Una aproximación narrativa a la construcción de la identidad laboral docente. Algunos aspectos biográficos previos a la formación inicial. Educación, Lenguaje y Sociedad, 4(4), 83-104. http://www.biblioteca. unlpam.edu.ar/pubpdf/ieles/n04a05mortola.pdf

Orozco, S. (2017). El saber profesional de una maestra de infantil. Revista Electrónica de Investigación Educativa, 19(2), 143-157. https://doi.org/10.24320/ redie.2017.19.2.1103

Pardo, M. \& Alderstein, C. (2016). Estrategia regional sobre docentes: Estado del arte y criterios orientadores para la elaboración de políticas de formación y desarrollo profesional de docentes de primera infancia en América Latina y el Caribe. OREALC-UNESCO.

París, G., Blanch, S., Comes, P., Edo, M., Estrada, J., Gimeno, X., Moron, M. \& Peire, T. (2017). Rúbrica d'autoavaluació de competències i habilitats personals i prosocials. Bellaterra: Dipòsit digital de documents de la UAB. http://ddd. uab.cat/pub/recdoc/2017/169417/RUBRICA_publicar_DDD.pdf

Pillen, M., Den Brok, P. \& Beijaard, D. (2013). Profiles and change in beginning teachers' professional identity tensions. Teaching and Teacher Education, 34, 86-97. https://doi.org/10.1016/j.tate.2013.04.003

Rivas-Flores, J. I. (2014). Narración frente al neoliberalismo en la formación docente. Visibilizar para transformar. magis, Revista Internacional de Investigación en Educación, 7(14), 99-112. https://doi.org/10.11144/Javeriana. M7-14.NFNF

Robinson, M. S. (2019). Construcción de la identidad profesional de la educadora de párvulos principiante en diferentes centros de educación infantil. Estudio de caso [Tesis de doctorado]. Universidad Autónoma de Barcelona. 
Robinson, M. S., Tejada, J. \& Blanch, S. (2018). ¿Cómo construyen su identidad las educadoras de párvulos principiantes? Una mirada desde diferentes realidades educativas. Perspectiva Educacional, 57(3), 104-130. http://perspec tivaeducacional.cl/index.php/peducacional/article/view/766

Stake, R. (2013). Estudios de casos cualitativos. En N. Denzin \& Y. Lincoln (Coords.), Manual de investigación cualitativa. Volumen III. Las estrategias de investigación cualitativa (pp. 428-444). Gedisa.

Sancho, J. M., Correa, J. M., Giró, X. \& Fraga, L. (Coords.) (2014). Aprender a ser docente en un mundo en cambio. Simposio internacional. Barcelona: Dipòsit Digital de la Universitat de Barcelona. http://hdl.handle.net/2445/50680

Tardif, M. \& Le Vasseur, L. (2018). Los profesores en Canadá. ¿Una identidad profesional en mutación? En M. Tardif \& I. Cantón (Coords.), Identidad profesional docente (pp. 19-32). Narcea.

Tejada, J., Carvalho-Días, M. L. \& Ruiz-Bueno, C. (2017). El prácticum en la formación de maestros: percepciones de los protagonistas. Magis. Revista Internacional de Investigación en Educación, 9(19), 91-114. https://doi. org/10.11144/Javeriana.m9-19.pfmp

Vergara, M. (2014). La construcción de la educadora infantil. Elementos para su comprensión. Pedagogía y Saberes, 41, 111-120. https://doi.org/10.17227/ 01212494.41 pys 111.120

Zapata, B. (2012). Metodología para el diseño curricular que promueve el desarrollo de competencias en la formación del educador/a de párvulos. En T. Goldrine (Ed.), Contextos y desafíos para la formación inicial del educador/a de párvulos (pp. 131-156). Ediciones Universitarias de Valparaíso. 